\title{
IMPACTO DE LA CONTAMINACIÓN AÉREA POR INCENDIOS FORESTALES EN LA MORBIMORTALIDAD DE LA POBLACIÓN EXPUESTA
}

\author{
IMPACT OF AIR POLLUTION BY FOREST FIRES AND THE MORBIDITY OF THE EXPOSED \\ POPULATION
}

\author{
Dra. Bárbara Sandoval D. ', Interna Medicina Dennise Rettig M. ${ }^{2}$, Dra. Tatiana Reyes R. ${ }^{3,5}$ y Dr. Manuel Oyarzún G. ${ }^{4,5}$ \\ 1.- Médico, becada de pediatría del Hospital San Juan de Dios de la Universidad de Chile. \\ 2.- Interna Medicina, Escuela de Medicina, Universidad de Chile. \\ 3.- Médico Internista y PhD, Profesora adjunta. \\ 4.- Neumólogo, Profesor titular. Miembro de número (sillónn³) Academia Chilena de Medicina. \\ 5.- Laboratorio de Investigaciones Respiratorias, Programa de Fisiopatología, ICBM, Facultad de Medicina, Universidad de Chile.
}

\section{INTRODUCCIÓN}

El riesgo sobre la salud en seres humanos atribuible de la contaminación atmosférica es conocido; siendo especialmente susceptibles los niños, embarazadas, ancianos y pacientes con patologías respiratorias y cardiovasculares (1). Además de la susceptibilidad y capacidad de adaptación de la población expuesta, los efectos de la contaminación atmosférica dependen de la composición de contaminantes, de sus eventuales sinergismos y de la dosis efectiva definida como el producto de tres factores: concentración del contaminante, tiempo de exposición y ventilación minuto, que resulta del producto de la frecuencia respiratoria por el volumen corriente (1).

Los incendios forestales afectan a bosques, sabanas y pastizales, emitiendo gases y partículas, pueden causar episodios de contaminación atmosférica relevantes. A diferencia de la contaminación atmosférica de origen urbano, la contaminación aérea de los incendios forestales puede recorrer grandes distancias, tiene una composición diferente y su ocurrencia es esporádica y más difícil de prever $(2,3)$.

En Chile los incendios forestales son favorecidos por condiciones meteorológicas estacionales: disminución en la humedad relativa del aire $(<30 \%)$, altas temperaturas $\left(>30^{\circ} \mathrm{C}\right)$ y vientos cálidos (>30 km/h) que en algunas temporadas son generados por la "corriente del niño" del océano Pacífico. A estos factores se suma el 'factor humano' $(99 \%$ de los incendios forestales son antropogénicos), la susceptibilidad a la ignición de los bosques y vegetación, y la compleja geografía nacional con zonas de difícil acceso (4).

Según la Corporación Nacional Forestal (CONAF) en Chile, en el periodo de un año (2019-2020) se produjeron 8127 incendios forestales que afectaron un total de 102.282
RESUMEN

La exposición a las emanaciones de incendios forestales es un importante problema de salud pública nacional e internacional. El cambio climático que conlleva sequía y aumento de la temperatura estival aumenta el riesgo y magnitud de los episodios de incendios forestales, generándose grandes incendios cuyas emanaciones pueden afectar a poblaciones distanciadas del epicentro. La asociación entre la exposición a las emanaciones de los incendios forestales, el aumento de las concentraciones de material particulado aéreo y la morbilidad respiratoria (exacerbación de asma y enfermedades respiratorias crónicas) ha sido evidenciada en diversos estudios. Sin embargo, es difícil realizar un metaanálisis de ellos, ya que la metodología empleada es muy disímil. Entre los principales mecanismos de morbilidad se encontrarían: la producción de citoquinas proinflamatorias, la activación endotelial y la disfunción del sistema nervioso autónomo. Ante la exposición al humo de incendios forestales, se produce daño tisular, aumento de los mecanismos protrombóticos, aumento de la presión arterial y cambios en el ritmo cardiaco, que explicaría los efectos cardiovasculares. Los sujetos con patología cardiovascular preexistente podrían tener mayor riesgo cardiovascular; sin embargo, existen factores confundentes en esta asociación. Por otra parte, el posible riesgo cancerígeno con la exposición a estas emanaciones requiere mayores estudios poblacionales.

Palabras claves: Incendios forestales; material particulado; salud pública; asma; enfermedades respiratorias; enfermedades cardiovasculares.

\section{ABSTRACT}

Exposure to forest fire fumes is a major national and international public health issue. Climate change that leads to drought and increased summer temperature increases the risk and magnitude of wildfires episodes, generating mega-fires whose fumes not only affect the boundary population, but they may become transcontinental. Association between exposure to forest fire fumes, mainly increased concentrations of air born particulate matter and respiratory morbidity (exacerbation of asthma and chronic respiratory diseases) has been evidenced by diverse studies. However, it is difficult to carry out meta-analysis with them since the methodology used is dissimilar. Among the main causes of morbidity have been postulated the production of proinflammatory cytokines, endothelial activation and dysfunction of the autonomic nervous system. Occurrence of tissue damage, increased prothrombotic mechanisms, increased blood pressure and changes in heart rate, would explain the cardiovascular effects associated with exposure to smoke from these fires. However, epidemiological outcomes have not been entirely consistent, as the association between cardiovascular morbidity and exposure to wildfire fumes may be mixed with confounding factors. Despite this, patients with pre-existing cardiovascular pathology may be at increased risk. Finally, the potential risk of carcinogen with exposure to these fumes requires further population studies.

Keywords: Wildfires: particulate matter; public health; asthma; respiratory disorders; cardiovascular diseases.

\section{Autor para correspondencia:}

Dr. Manuel Oyarzún Gómez

Laboratorio de Investigaciones Respiratorias - Instituto de Ciencias Biomédicas.

Facultad de Medicina, Universidad de Chile.

Avda. Salvador 486. Comuna de Providencia - Santiago, Chile (Teléfono: 222266893)

Email:manueloyarzun@uchile.cl 
hectáreas (5), o sea el equivalente a 1,6 veces la superficie de la ciudad de Santiago. En el último decenio (2011-2020) la CONAF registra un promedio anual de 6398 incendios, afectando anualmente a 122.328 ha equivalente a 1,9 veces el área de Santiago (Figura 1).

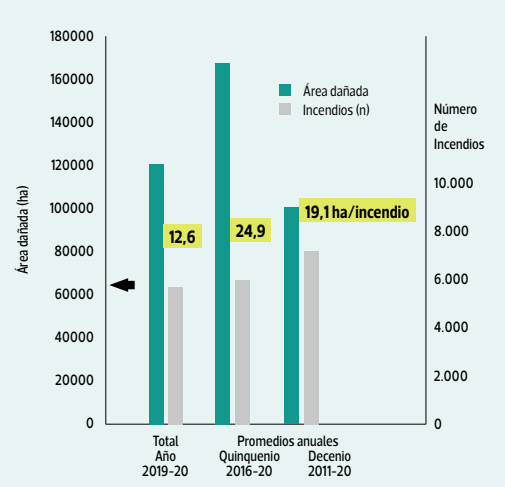

Figura 1. Ocurrencia y área dañada en Chile por incendios forestales en el decenio 2011-2020. $(\leftarrow)$ indica la superficie total de la ciudad de Santiago (64.100 ha). Gráfico elaborado por los autores con datos de la CONAF (5).

Las regiones de Chile más afectadas en el último quinquenio son Bío-Bío, Araucanía, Maule y Valparaíso. Con una pérdida directa anual equivalente a 50 millones de USD, suma que no incluye pérdidas en infraestructura y puestos de trabajo, gastos en salud de la población expuesta y su impacto ecológico. (5).

Su efecto ecológico más notable, es su contribución al cambio climático a través de la gran producción de gases de efecto invernadero como el dióxido de carbono $\left(\mathrm{CO}_{2}\right)(6)$. Se estima que la emisión de $\mathrm{CO}_{2}$ global ha aumentado 3 veces su valor: desde 0,4 Gigatonelada anual en los '90 alcanzó 1 Gigatonelada anual entre 2011 y 2015 (7). Estos incendios provocan también pérdida de biomasa, alteración de los suelos (v.gr. erosión), deterioro de los recursos hídricos (v.gr. contaminación del agua) y afectan la biodiversidad (v.gr. alteración de los hábitats y daños en la flora y fauna nativa) (8).

Los impactos socioeconómicos de los incendios forestales son también importantes ya que incluyen daño de la propiedad pública y privada, como pérdida de viviendas, parques y reservas nacionales, disminución de fuentes laborales (pérdida de industrias y cultivos) y además afectan la salud de la población al aumentar la morbimortalidad por enfermedades respiratorias y cardiovasculares (8)

A pesar de la magnitud y del alto número de incendios forestales que afectan anualmente a nuestro país, no existen estudios nacionales sobre cómo estos afectan la salud de la población expuesta.

En esta revisión bibliográfica en PubMed, SciELO y Google que abarca desde 1983 hasta 2019 analizamos el aumento de la contaminación atmosférica durante los incendios forestales con vegetación similar a la nacional -excluyendo los incendios de arrozales-, y sus efectos deletéreos para la salud de la población humana expuesta a las emanaciones de estos incendios. Usando los términos 'contaminantes atmosféricos', 'incendios forestales', 'efectos cardiovasculares' y 'cáncer' se obtuvo una base inicial de 100 publicaciones en inglés y español, de las cuales seleccionamos veinte.

\section{Componentes de las emisiones de los incen- dios forestales}

Los incendios forestales son parte del ciclo del carbono terráqueo, ya que contribuyen en la circulación del carbono terrestre a la atmósfera.

Las emisiones de los otros componentes del humo de incendios forestales dependen del tipo de vegetación y de las condiciones meteorológicas. Carecemos de modelos teóricos para predecir las características de los productos de combustión, y la información deriva de mediciones en diversos territorios afectados por incendios forestales (3).

La composición del humo de los incendios forestales varía según la fase de la combustión. En la fase de flama emite $\mathrm{CO}_{2}$, monóxido de carbono (CO), dióxido de azufre $\left(\mathrm{SO}_{2}\right)$, óxido nítrico (NO) y dióxido de nitrógeno $\left(\mathrm{NO}_{2}\right)$. Al extinguirse la llama aumenta la concentración de gases (v. gr. NO) y en la etapa de brasas se emite más $\mathrm{CO}_{\text {y }} \mathrm{CO}_{2}(3)$.

Resumiendo, estudios en diversos países del orbe han mostrado que los componentes del humo de los incendios forestales y fuegos agrícolas incluyen además de $\mathrm{CO}_{2}, \mathrm{CO}$ y material particulado, compuestos orgánicos volátiles (COVs) (hidrocarburos aromáticos policíclicos-HAPs, benceno, aldehídos, fenoles) y gases inorgánicos como óxidos de nitrógeno $(N O x)(9,10)$. Además, la calidad del aire es afectada por la generación de contaminantes secundarios como aerosoles orgánicos y ozono generados por la reacción de NOx y COVs con radiación ultravioleta. (11).

\section{Riesgos para la salud humana}

Los impactos de la exposición a las emanaciones de los incendios forestales incluyen efectos subclínicos que son asintomáticos y efectos clínicos que producen morbilidad, consultas, días de inactividad, hospitalizaciones y eventualmente mortalidad (Figura 2).

En esta revisión nos referiremos a los efectos respiratorios, cardiovasculares y cancerígenos de la contaminación por incendios forestales y sus efectos en poblaciones específicas.

\section{Mecanismos del daño respiratorio y cere- bro-cardiovascular del material particulado (PM)}

Los mecanismos fisiopatológicos propuestos para explicar la morbilidad respiratoria y cardiovascular asociada a la exposición de material particulado aéreo proveniente de incendios forestales se inician en el tejido pulmonar. En el pulmón se liberan citoquinas, como Interleuquina 1 beta (IL -1ß) e Interleuquina 6 (IL-6), que promueven una respuesta inflamatoria pulmonar y sistémica (hígado y médula ósea). Además se produce disfunción endotelial, asociado a hipercoagulabilidad san-

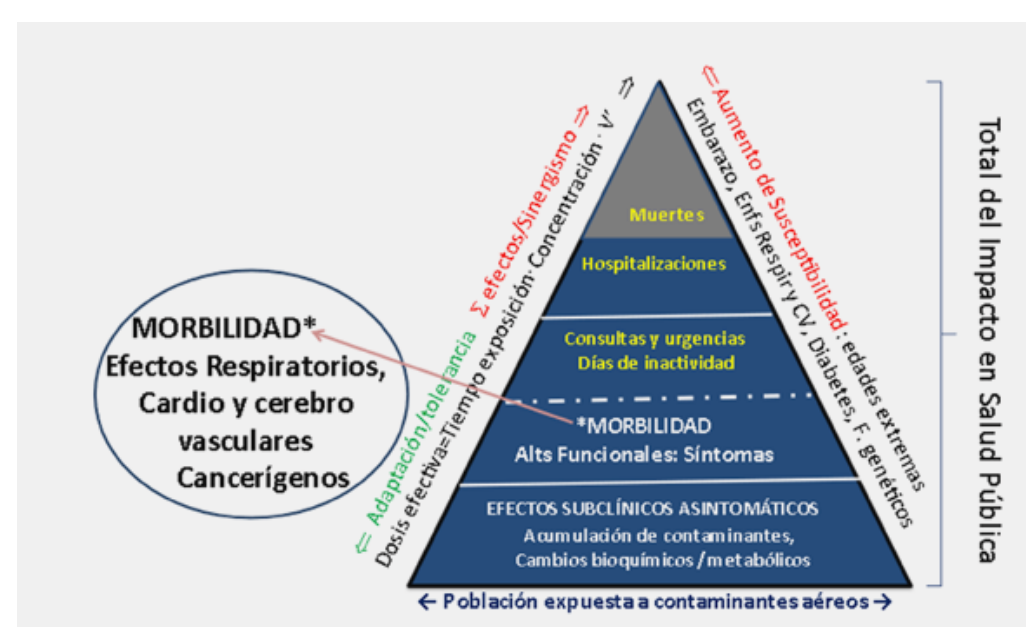

Figura 2. Impactos de la contaminación aérea por las emanaciones de los incendios forestales en la salud de la población expuesta. Alts.: alteraciones; V': ventilación minuto; Enfs Respir y CV: Enfermedades respiratorias y cardiovasculares. F. Genéticos: Factores genéticos. (12) 
guínea por daño endotelial y a aumento de plaquetas, viscosidad sanguínea y fibrinógeno. La generación de estrés oxidativo, por liberación de especies reactivas del oxígeno y oxidación de lípidos sanguíneos y estimulación de receptores, provoca disminución del tono vagal, aumento de la presión arterial y alteraciones del ritmo cardíaco. Estos se resumen en tres mecanismos: efectos proinflamatorios del endotelio pulmonar, difusión directa de partículas ultrafinas (PM0,1) través de la barrera alvéolo-capilar y reflejos autonómicos por activación neuronal pulmonar (3,11) (Figura 3).

Estudios 'in vivo' de $\mathrm{SO}_{2}$ y $\mathrm{PM}$ de incendios forestales detectaron un nucleósido generado por oxidación de ADN, la 8-0xo-2' deoxiguanosina, la cual se puede medir en la orina y es un biomarcador de cáncer, siendo un eventual mecanismo de cuadros oncológicos asociados a incendios forestales (3). Por otra parte, la exposición ocupacional de bomberos de incendios forestales aumentó entre 1,83 y 4,23 veces las concentraciones urinarias pre-exposición de 9 especies de PAHs hidroxilados producidos por oxidación de los PAHs inhalados, algunos de los cuales pueden tener efectos mutagénicos y/o carcinogénicos (13).

\section{Efectos sobre la morbilidad respiratoria}

El material particulado aéreo (PM) es el contaminante que más aumenta en zonas de incendios forestales, superando las concentraciones basales urbanas. El humo puede recorrer grandes distancias y alcanzar ciudades generando un problema de salud pública por aumento de PM (3). Los escasos estudios comparativos sugieren que el PM10 de incendios forestales es al menos igualmente tóxico que el urbano (3). El aumento de PM durante incendios forestales incrementa las consultas por enfermedades respiratorias, hasta cinco semanas después del inicio del incendio (14). Además, aumenta las admisiones hospitalarias respiratorias, dentro de ellas las exacerbaciones de asma y enfermedad pulmonar obstructiva crónica (EPOC), correlacionándose con la elevación de las concentraciones de PM10 (15).

En diferentes territorios se ha demostrado una asociación positiva entre el aumento de las concentraciones de PM2.5 y el aumento de consultas respiratorias, especialmente por asma en población adulta. Además, el aumento del PM2.5 se asocia al desarrollo de bronquitis, bronquiolitis y neumonía (2). (Tabla 1)

El aumento del PM durante los incendios forestales exacerba el asma y rinitis y también induce irritación y prurito ocular, por alteración de la película del lagrimal y aumento de la presión de la conjuntiva bulbar (16).

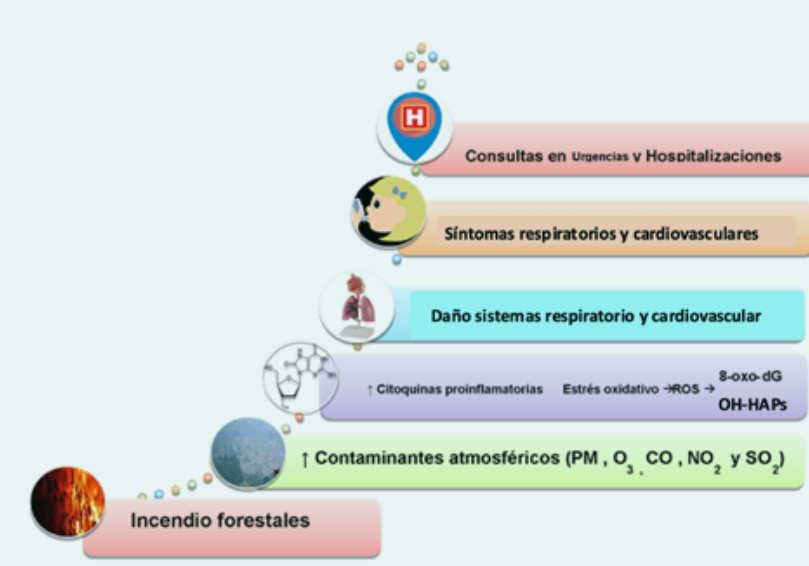

Figura 3. Principales mecanismos propuestos en la generación del daño respiratorio y cardiovascular de la contaminación aérea de los incendios forestales y sus consecuencias en salud. Citoquinas proinflamatorias: especialmente interleuquinas (IL-1ß e IL6) liberadas por el tejido pulmonar, hígado y médula ósea. $\uparrow$ : aumento; $\mathrm{PM}$ : material particulado; $\mathrm{O}_{3}$ : ozono; $\mathrm{CO}$ : monóxido de carbono; $\mathrm{NO}_{2}$ : dioxido de nitrógeno; $\mathrm{SO}_{2}$ : dióxido de azufre; ROS: especies reactivas de oxígeno; 8-oxo-dG: 8-0xo-2'desoxiguanosina; OH-HAPs: hidrocarburos aromáticos policíclicos hidroxilados.

Tabla 1. Estudios sobre efectos respiratorios de la exposición a material particulado aéreo proveniente de incendios forestales.

\begin{tabular}{|c|c|c|}
\hline Estudio y país & Exposición & Resultados \\
\hline $\begin{array}{l}\text { Chen et al (2006) } \\
\text { (Brisbane, } \\
\text { Australia) }{ }^{15}\end{array}$ & $\begin{array}{l}\text { PM10, promedio diario por } \\
\text { hora: }>50 \mu \mathrm{g} / \mathrm{m}^{3}\end{array}$ & $\begin{array}{l}\text { A concentraciones PM10 }>20 \mu \mathrm{g} / \mathrm{m}^{3} \text { vs concentraciones > } \\
15 \mu \mathrm{g} / \mathrm{m}^{3} \text { aumenta las admisiones hospitalarias de causa } \\
\text { respiratoria. OR: } 19 \% \text { (IC: } 9 \%-30 \%)\end{array}$ \\
\hline $\begin{array}{l}\text { Johnston et al. } \\
\text { (2006) (Aus- } \\
\text { tralia) }{ }^{17}\end{array}$ & $\begin{array}{c}20 \mu \mathrm{g} / \mathrm{m}^{3} \text { promedio anual } \\
50 \mathrm{ug} / \mathrm{m}^{3} \text { promedio día }\end{array}$ & $\begin{array}{c}\text { A concentraciones > } 10 \mu \mathrm{g} / \mathrm{m}^{3} \text { aumentan los síntomas } \\
\text { respiratorios en asmáticos adultos y niños } \\
\text { OR: } 24 \%(\mathrm{IC}: 11 \%-39 \%)\end{array}$ \\
\hline $\begin{array}{l}\text { Johnston et al. } \\
\text { (2007) (Darwin, } \\
\text { Australia) }{ }^{18}\end{array}$ & $\begin{array}{c}40 \mu \mathrm{g} / \mathrm{m}^{3} \mathrm{promedio} \text { anual } \\
200 \mathrm{ug} / \mathrm{m}^{3} 1 \mathrm{~h} \text { max }\end{array}$ & $\begin{array}{c}\text { Aumento }>10 \mu / \mathrm{m}^{3} \text { de PM10 no aumentan significativamente } \\
\text { las hospitalizaciones de causa respiratoria OR } 1,08 \\
\text { (IC: } 0,98-1,18) \\
\text { Aumentan consultas por EPOC } \\
\text { OR }=1,21 \text { (IC:1,00-1,47) }\end{array}$ \\
\hline $\begin{array}{l}\text { Hanigan et al. } \\
\text { (2008) (Darwin, } \\
\text { Australia) }{ }^{19}\end{array}$ & $\begin{array}{l}\text { PM10, promedio diario: } \\
21 \mu \mathrm{g} / \mathrm{m}^{3}\end{array}$ & $\begin{array}{l}\text { Aumento }>10 \mu \mathrm{g} / \mathrm{m}^{3} \mathrm{PM} 10 \text { asociación }(+) \text { con } \\
\text { hospitalizaciones de causa respiratoria } \\
\text { OR }=4,81 \%(\text { IC:-1,10-11,01) }\end{array}$ \\
\hline $\begin{array}{l}\text { Tham et al. } \\
\text { (2009) (Victoria, } \\
\text { Australia) }\end{array}$ & PM10, peor día >288 $\mu \mathrm{g} / \mathrm{m}^{3}$ & $\begin{array}{c}\text { A concentraciones de PM10 } 9.1 \mu \mathrm{g} / \mathrm{m}^{3} \text { aumentan los síntomas } \\
\text { respiratorios } \\
\text { OR }=1,8 \%(\mathrm{IC}: 0,4-3,3 \%) \\
\text { Sin asociación con admisiones hospitalarias por esta causa. }\end{array}$ \\
\hline $\begin{array}{l}\text { Hutchinson et al. } \\
\text { (2007) (Estados } \\
\text { Unidos) }{ }^{21}\end{array}$ & $\begin{array}{c}\text { PM2,5 mayor concentración } \\
283,9 \mu \mathrm{g} / \mathrm{m}^{3}\end{array}$ & $\begin{array}{c}\text { A concentraciones de } \mathrm{PM} 2.5>35 \mu \mathrm{g} / \mathrm{m}^{3} \text { y tras } 48 \mathrm{~h} \text { de expo- } \\
\text { sición hay asociación significativa con consultas de asma en } \\
\text { servicios de urgencia. } \\
\text { OR }=1.51 \text { ( IC: } 1,00-2,228)\end{array}$ \\
\hline $\begin{array}{l}\text { Delfino et al. } \\
(2009) \text { (Estados } \\
\text { Unidos) }{ }^{22}\end{array}$ & $\begin{array}{c}\text { PM2,5 mayor concentración } \\
240 \mu \mathrm{g} / \mathrm{m}^{3}\end{array}$ & $\begin{array}{l}\text { Aumento ingresos hospitalarias por asma en adultos } \\
\text { entre } 65 \text { y } 69 \text { años } \\
\text { OR }=10,1(\text { IC: } 3,0-17,8) \text {, y niños } 0 \text { a } 4 \text { años } \\
\text { OR= } 8,3(\text { IC: } 2,2-14,9)\end{array}$ \\
\hline $\begin{array}{l}\text { Yao et al. (2020) } \\
\text { (Canadá) }\end{array}$ & $\begin{array}{c}\text { PM2,5 a concentraciones } \\
10 \mu \mathrm{g} / \mathrm{m}^{3}\end{array}$ & $\begin{array}{l}\text { Se observó aumento de consultas en urgencias } \\
\text { por asma y EPOC } \\
\text { OR }=1,098 \text { (IC: } 1,009-1,067)\end{array}$ \\
\hline $\begin{array}{l}\text { Vicedo- Cabrera } \\
\text { et al. (2016) } \\
\text { (España) }^{24}\end{array}$ & $\begin{array}{c}\text { PM10 mayor concentración } \\
175 \mu \mathrm{g} / \mathrm{m}^{3}\end{array}$ & $\begin{array}{l}\text { Mayor riesgo de prurito ocular y lagrimeo en niños asmáticos } \\
\text { OR 9,26 ( IC : } 2,14-40,12 \text { ) y con rinitis OR } 8,06 \text { (IC: } 1,98 \text { - } \\
32,88 \text { ) vs población no asmática OR=3,23 (IC: } 1,63-6,4) \text { y sin } \\
\text { rinitis } 0 R=3,23 \text { (IC:1,58 -6,59) }\end{array}$ \\
\hline
\end{tabular}

OR: odds ratio ; IC: intervalo de confianza 95\%; PM: material particulado, subíndice 10 y 2,5 indica que el diámetro aerodinámico de las partículas es $\leq 10 y \leq 2,5 \mu \mathrm{m}$. respectivamente. 
Resumiendo, PM (especialmente PM2,5), $\mathrm{SO}_{2}$ y NOx de incendios forestales aumentan. la morbilidad respiratoria de la población expuesta provocando: exacerbación de enfermedades respiratorias preexistentes -como asma y rinitis-, aumento de consultas por asma y eventualmente bronquitis, bronquiolitis y neumonía.

\section{Efectos respiratorios en población infantil}

La población infantil expuesta a altas concentraciones de PM2,5 de incendios forestales, registra más ingresos hospitalarios respiratorios en lactantes y preescolares, esto no se observa en escolares ni adolescentes $(25,26)$. En niños de 0-5 años expuestos a emanaciones de incendios forestales en San Diego, California, también hubo aumento de admisiones hospitalarias y ese rango etario registró mayor número de consultas por enfermedades respiratorias (25).

El aumento de consultas respiratorias asociados a la exposición de PM2,5, no se acompaña de disminución de la función pulmonar en niños asmáticos de 4-11 años; esta disminución sí se ha detectado en mayores de 12 años (26).

Además, la exposición al humo de incendios forestales, aumenta el uso de agonistas $\beta-2$ adrenérgicos de acción corta en niños asmáticos obesos versus niños asmáticos con normo peso y sobrepeso, aunque no se evidenció aumento de consultas en servicios de urgencias, hospitalizaciones, ni nuevos diagnósticos de asma (27).

De los efectos de gases originados en incendios forestales en niños, el $\mathrm{SO}_{2}$ genera inflamación del tracto respiratorio, haciendo más susceptibles a los niños a infecciones respiratorias. La exposición a NOx se asocia a síntomas de asma e irritación de vías respiratorias, aumentando las consultas y hospitalizaciones.. Los NOx contribuyen a la formación de $\mathrm{O}_{3}$, el cual se asocia, a exacerbación del asma y disminución de la función pulmonar. Sin embargo, en población infantil del sudeste asiático no se evidenció una asociación positiva con $\mathrm{O}_{3}$ entre la exposición de humo y sibilancias, aunque sí se encontró esta asociación con $\mathrm{SO}_{2}$ y NO 2 y también con PM10 y PM2,5 (28).

\section{Efectos en la morbilidad cardiovascular}

Estudios australianos señalan que el aumento del PM atmosférico urbano, aumenta el número de ingresos por enfermedades cardiovasculares en mayores de 65 años, principalmente por infarto agudo de miocardio y en sujetos jóvenes se encuentra asociado con arritmias (29). Sin embargo, la evidencia no es totalmente concordante, existiendo estudios que no encuentran asociación entre exposición e ingresos hospitalarios por causa cardiovascular y otros que síla demuestran. $(10,18,29,30)$.

Además de los contaminantes, hay otros factores ambientales que acentúan la aparición de problemas cardiovasculares en los incendios forestales que deben considerarse al analizar los resultados, entre ellos la humedad relativa $(10,14,29)$, la temperatura $(10,14)$, días lluviosos (14) y días festivos $(10,14,30)$.

Resumiendo, las evidencias de asociación entre enfermedades cardiovasculares y la contaminación aérea (CO y PM) de incendios forestales están mezcladas con factores confundentes y por ello no son totalmente concluyentes. Siendo factible que la preexistencia de enfermedades cardiovasculares implique mayor riesgo (Tabla 2).

\section{Efectos cancerígenos}

En el humo hay más de 15 COPCs (Chemicals of Potential Concern) considerados potencialmente peligrosos para la salud. Sin embargo, sólo benceno, formaldehído, acroleína y PM3,5 alcanzarían altas concentraciones durante un incendio de biomasa (31). El formaldehído, considerado cancerígeno por la Agencia Internacional para Investigación en Cáncer (IARC), puede causar cáncer nasofaríngeo, y está asociado con leucemia (32). El formaldehido y el acetaldehído se relacionan con cáncer, asma y alteraciones del epitelio nasal (33).
Estos aldehídos son emitidos directamente por sus fuentes ( $\mathrm{v}$.gr. quema de madera) existiendo estudios que relacionan aumento del riesgo de cáncer con exposición a incendios forestales donde aumentan las concentraciones de formaldehido y acetaldehído. (34).

\section{CONCLUSIONES}

La exposición a emanaciones de incendios forestales es un problema creciente de salud pública tanto en Chile como en el resto del mundo.

Existe asociación entre exposición a PM de los incendios forestales y mortalidad global y morbilidad respiratoria por Asma, EPOC y Neumonía. En > 65 años esta exposición aumenta las consultas por enfermedades respiratorias agudas y exacerba enfermedades respiratorias crónicas (RR=1,19 IC: 1,09-1,30).

En la población infantil los asmáticos son más susceptibles a la exposición de PM durante los incendios forestales, siendo más afectados los niños entre 0 a 4 años $O R=8,3 \%$ (IC 95\% 2,2-14,9) y los obesos serían más susceptibles

Las evidencias de asociación entre enfermedades cardiovasculares y contaminación aérea (CO \& PM) de incendios forestales están mezcladas con factores confundentes,

Tabla 2. Estudios sobre efectos cardiovasculares de la exposición a material particulado aéreo proveniente de incendios forestales.

\begin{tabular}{|c|c|c|c|}
\hline $\begin{array}{l}\text { Estudio y } \\
\text { país }\end{array}$ & $\begin{array}{c}\text { Variable } \\
\text { independiente }\end{array}$ & $\begin{array}{c}\text { Variable } \\
\text { dependiente }\end{array}$ & $\begin{array}{c}\text { Morbilidad } \\
\text { Cardiovascular }\end{array}$ \\
\hline $\begin{array}{l}\text { Johnston } \\
\text { et al 2007, } \\
\text { Australia }{ }^{18}\end{array}$ & $\begin{array}{l}\text { PM } 10 \text { en tres tem- } \\
\text { poradas de incendios } \\
\text { forestales (2000, } \\
2004 \text { y 2005) }\end{array}$ & $\begin{array}{l}\text { Admisiones hospitalarias } \\
\text { por cardiopatía isquémica }\end{array}$ & $\begin{array}{c}\text { Asociación positiva con la cardiopatía } \\
\text { isquémica en los pueblos indígenas } \\
\text { OR }=1,71(\text { IC: } 1,14-2,55)\end{array}$ \\
\hline $\begin{array}{l}\text { Barnett et al } \\
2006, \text { Aus- } \\
\text { tralia y Nueva } \\
\text { Zelandia }{ }^{29}\end{array}$ & $\begin{array}{l}\text { Contaminantes: } \\
\text { - CO } \\
\text { PM2,5 } \\
\text { - ozono }\end{array}$ & $\begin{array}{l}\text { Admisiones hospitalarias } \\
\text { cardiovasculares v,gr. } \\
\text { cardiopatías isquémicas }\end{array}$ & $\begin{array}{l}\uparrow \text { CO aumenta los ingresos hospita- } \\
\text { larios de ancianos por enfermedad } \\
\text { cardiovasculares totales } \mathrm{OR}=1,3 \\
\text { (IC: } 0,6-2)\end{array}$ \\
\hline $\begin{array}{l}\text { Haikerwal } \\
\text { et al, } 2015 \text {, } \\
\text { Australia } 30\end{array}$ & $\begin{array}{l}\text { PM2,5 durante Incen- } \\
\text { dios forestales ( } 2006 \\
\text { y 2007) en Victoria, } \\
\text { Australia }\end{array}$ & $\begin{array}{l}\text { Admisiones hospitalarias } \\
\text { cardio-vasculares. Cardio- } \\
\text { patia isquémica }\end{array}$ & $\begin{array}{c}\uparrow \text { Riesgo de paro cardíaco extra- } \\
\text { hospitalario } \\
\text { OR }=6,98 \% \text { (IC: } 1,03-13,29) \\
\uparrow \text { Riesgo de asistencia a emergencia } \\
\text { por Cardiopatía isquémica } \\
\text { OR } 1,86 \% \text { (IC } 0,35-3,4 \%)\end{array}$ \\
\hline $\begin{array}{l}\text { Dennekamp } \\
\text { et al, } 2015 \text {, } \\
\text { Australia }{ }^{10}\end{array}$ & $\begin{array}{l}\text { PM2,5 PM10 y C0 } \\
\text { durante incendios } \\
\text { forestales ( } 2006 \text { y } \\
\text { 2007) en Melbourne, } \\
\text { Australia }\end{array}$ & $\begin{array}{c}\text { Atenciones en ambulancia } \\
\text { por paro cardiaco extra } \\
\text { hospitalario }\end{array}$ & $\begin{array}{c}\uparrow \text { Hospitalizaciones cardiacas con } \uparrow \\
\text { PM } 2,56,1 \mu \mathrm{g} / \mathrm{m}^{3} \\
\text { OR } 8,05 \%(\mathrm{Cl}: 2.30-14,13) \uparrow \mathrm{CO} \\
\text { de } 0,3 \mathrm{ppm} \\
\text { OR }=35,7 \%(\mathrm{Cl}: 8,98-68,92)\end{array}$ \\
\hline $\begin{array}{l}\text { Faustini et al, } \\
\text { 2015, Sur de } \\
\text { Europa }^{9}\end{array}$ & $\begin{array}{l}\text { PM10 proveniente de } \\
\text { incendios forestales } \\
(2003-2010)\end{array}$ & Mortalidad & $\begin{array}{l}\text { Aumento de la mortalidad } \\
\text { cardiovascular } \\
\text { OR=6,29\% (IC: } 1,00-11,85)\end{array}$ \\
\hline
\end{tabular}

OR: odds ratio ; IC: intervalo de confianza 95\%; PM: material particulado, subíndice 10 y 2,5 indica que el diámetro aerodinámico de las partículas es $\leq 10 \mathrm{y} \leq 2,5 \mu \mathrm{m}$. respectivamente. 
no siendo totalmente concluyentes. Por otra parte, el posible riesgo cancerígeno de la exposición a estas emanaciones requiere mayores estudios

Los portadores de enfermedades respiratorias crónicas y posiblemente cardiovasculares, senescentes, embarazadas y niños son las personas con mayor riesgo.

Concordamos con Black et al (35) en que los vacíos más relevantes en este tema son los efectos a largo plazo y la recuperación de la exposición a las emanaciones de incendios forestales y las consecuencias de esta exposición en la salud infantil.

\section{Los autores declaran no tener conflictos} de interés.

\section{REFERÉNCIAS}

1. Oyarzún M, Pino P. Contaminación atmosférica y del aire intradomiciliario En: Enfermedades Respiratorias en Pediatría Eds. O. Herrera y A Quezada. Editorial Mediterráneo Ltda. 3ả Edición. Santiago de Chile, 2012. Págs. 39-48.

2 Dennkamp M: Abramson M. The effects of bushfire smoke on respiratory health. Respirology, 2011,16 (2): 198-209. doi: 10.1111/j.1440-1843.2010.01868.x. PMID 20920143.

3. Sandoval B, Reyes T, Oyarzún M. Mecanismos de los efectos nocivos para la salud de la contaminación atmosférica proveniente de incendios forestales. Rev Chil Enferm. Respir. 2019, 35(1): 49-57

4. Peña-Fernández, E; Valenzuela-Palma, L. Incremento de los incendios forestales en bosques naturales y plantaciones forestales en Chile. En Memorias del segundo simposio internacional sobre políticas, planificación y economía de los programas de protección contra incendios forestales: Una visión global. 2008. p. 595-612. Disponible en: https://www.fs.fed.us/psw/publications/ documents/psw_gtr208es/psw_gtr208es_595-612 pena-fernandez.pdf (Fecha de consulta:10 .05.2021).

5. 5. CONAF. Número de incendios forestales y superficie afectada a la fecha. Disponible en: https://www.conaf. $\mathrm{cl} /$ incendios-forestales/incendios-forestales-en-chile/estadisticas-historicas/ //Fecha de consulta 06.05.2021)

6. Chankina O V, Churkina TV, Ivanov A V, Ivanov VA, Ivanova CA. Multielement composition of the aerosols of the forest fires of boreal forests upon burning of forest combustibles. Nuclear Instruments and Methods in Physics Research. 2001;470:444-447.
7. Federici S, Tubiello F, Salvatore M, Jacobs H, Schmidhuber J. Forest Ecology Managemwnt New estimates of $\mathrm{CO}_{2}$ forest emissions and removals: $1990-2015 \mathrm{q}$ For Ecol Manage. 2015;352:89-98. doi:10.1016/j.foreC0.2015.04.022.

8. Castillo $M$, Pedernera P, Peña E. Incendios forestales y medio ambiente : una síntesis global. Revista ambiente y desarrollo de CIPMA.2003;19 (1):44-53.

9. Faustini A, Alessandrini ER, Pey J, Perez N, Samoli E, Querol $X$, et al MED-PARTICLES study group. Short-term effects of particulate matter on mortality during forest fires in Southern Europe: results of the MED-PARTICLES Project. Occup Environ Med. 2015;72(5):323-9. doi: 10.1136/oemed-2014-102459.

10. Dennekamp, M., Straney, L. D., Erbas, B., Abramson, M. J., Keywood, M., Smith, K., et al. Forest Fire Smoke Exposures and Out-of-Hospital Cardiac Arrests in Melbourne, Australia: A Case-Crossover Study. Environ Health Perspect 2015, 123(10), 959-964. doi: 10.1289/ehp.1408436.

11. Cascio, W. Wildland fire smoke and human health. Sci Total Environ 2018, 624:, 586-95. doi: 10.1016/j.scitotenv.2017.12.086

12. Oyarzún M. Efectos para la salud de la contaminación aérea proveniente de los incendios forestales. Boletín Academia Chilena de Medicina. 2020. 57: 84-8.

13. Adetona O, Simpson CD, Li Z, Sjodin A, Calafat AM, Naeher LP. Hydroxylated polycyclic aromatic hydrocarbons as biomarkers of exposure to wood smoke in wildland firefighters. J Expo Sci Environ Epidemiol. 2017;27(1):7883. doi: 10.1038/jes.2015.75

14. Moore D, Copes R, Fisk R, Joy R, Chan K, Brauer M. Population health effects of air quality changes due to forest fires in British Columbia in 2003: estimates from physician-visit billing data. Can I Public Health 2006;97(2):105-108. doi: 10.1007/BF03405325.

15. Chen $\mathrm{L}$, Verrall $\mathrm{K}$, Tong $\mathrm{S}$. Air particulate pollution due to bushfires and respiratory hospital admissions in Brisbane, Australia. Int J Environ Health Res. 2006 Jun;16(3):18191. doi:10.1080/09603120600641334.

16. Verdejo L, Juarez M, Sala B. Contingencia ambiental por contaminación atmosférica y su asociación con síntomas y signos respiratorios y oculares. Salud en Tabasco 2000 6(001), 299-301. Disponible en: http://redalyc.uaemex $\mathrm{mx} / \mathrm{src} /$ inicio $/$ ArtPdfRed.jsp?iCve=48760103\&iC veNum=2691

17. Johnston FH, Webby RJ, Pilotto LS, Bailie RS, Parry DL, Halpin SJ. Vegetation fires, particulate air pollution and asthma: a panel study in the Australian monsoon tropics. Int J Environ Health Res. 2006 Dec;16(6):391-404. doi: 10.1080/09603120600641334.

18. Johnston F, Bailie R, Pilotto L, Hanigan I. Ambient biomass smoke and cardio-respiratory hospital admissions. in Darwin, Australia. BMC Public Health 7, 240 (2007) doi:10.1186/1471-2458-7-240

19. Hanigan IC, Johnston FH, Morgan GG. Vegetation fire smoke, indigenous status and cardio-respiratory hospital admissions in Darwin, Australia, 1996-2005: a time-series study. Environ Health. 2008 Aug 5;7:42. doi: 10.1186/1476-069X-7-42

20. Tham R, Erbas B, Akram M, Dennekamp M, Abramson MJ. The impact of smoke on respiratory hospital outcomes during the 2002-2003 bushfire season, Victoria, Australia. Respirology. 2009 Jan;14(1):69-75. doi: 10.1111/j.14401843.2008.01416.x

21. Hutchinson, J. A., Vargo, J., Milet, M., French, N., Billmire, M. Johnson, J., \& Hoshiko, S. (2018). The San Diego 2007 wildfires and Medi-Cal emergency department presentations, inpatient hospitalizations, and outpatient visits: An observational study of smoke exposure periods and a bidirectional case-crossover analysis. PLoS medicine, 15(7), e1002601. https://doi.org/10.1371/journal. pmed.1002601

22. Delfino RJ, Brummel S, Wu J, Stern H, Ostro B, Lipsett M, et al. The relationship of respiratory and cardiovascular hospital admissions to the southern California wildfires of 2003 Occup Environ Med. 2009 Mar;66(3):189-97. doi: 10.1136/ oem.2008.041376

23. 23. Yao J, Brauer M, Wei J, McGrail KM, Johnston FH, Henderson SB. Sub-Daily Exposure to Fine Particulate Matter and Ambulance Dispatches during Wildfire Seasons: A Case-Crossover Study in British Columbia, Canada. Environ Health Perspect. 2020 Jun;128(6):67006. doi: 10.1289/ EHP5792.

24. Vicedo-Cabrera, A. M., Esplugues, A., Iñíguez, C., Estarlich, M., Ballester, F. Health effects of the 2012 Valencia (Spain) wildfires on children in a cohort study. Environ Geochem Health. $2016 ， 38(3), 703-712$. https://doi: 10.1007/ s10653-015-9753-5.

25. Leibel S, Nguyen M, Brick W, Parker J, llango S, Aguilera R et al Increase in Pediatric Respiratory Visits Associated with Santa Ana Wind-Driven Wildfire Smoke and PM2.5 Levels in San Diego County. Ann Am Thorac Soc. 2020 Mar; 17(3):313320. doi: 10.1513/AnnalsATS.201902-1500C. PMID: 31860802.

26. Lipner EM, O'Dell K, Brey SJ, Ford B, Pierce JR, Fischer EV, Crooks IL. The Associations Between Clinical Respiratory Outcomes and Ambient Wildfire Smoke Exposure Among Pediatric Asthma Patients at National Jewish Health, 2012-2015. Geohealth. 2019 3:3(6):146-159 doi: 10.1029/2018CH000142. PMID: 32159037; PMCID: PMC7007069.

27. Tse K, Chen L, Tse M, Zuraw B, Christiansen S. Effect of catastrophic wildfires on asthmatic outcomes in obese children: breathing fire. Ann Allergy Asthma Immunol. 2015 Apr;114(4):308-311.e4. doi: 10.1016/j.anai.2015.01.018. Epub 2015 Mar 4. PMID: 25747784; PMCID: PMC4387007.

28. Luong LMT, Sly PD, Thai PK, Phung D. Impact of ambient air pollution and wheeze-associated disorders in children in Southeast Asia: a systematic review and meta-analysis. Rev Environ Health. 2019 Jun 26;34(2):125-139. doi: 10.1515/ reveh-2018-0079. PMID: 30753165.

29. Barnett AG, Williams GM, Schwartz J, Best TL, Neller AH, Petroeschevsky AL et al The Effects of Air Pollution on Hospitalizations for Cardiovascular Disease in Elderly People in Australian and New Zealand Cities. Environ Health Perspect; 2006;1018(7):1018-1023. doi:10.1289/ehp.8674.

30. Haikerwal A, Akram M, Del Monaco A, Smith K, Sim MR, Meyer M et al Impact of Fine Particulate Matter (PM2.5) Exposure During Wildfires on Cardiovascular Health Outcomes. J Am Heart Assoc. 2015 Jul 15:4(7):e001653 doi: 10.1161/ JAHA.114.001653.PMID: 26178402; PMCID: PMC4608063.

31. Booze T, Reinhardt T, Quiring S, Ottmar RD. A Screening-Level Assessment of the Health Risks of Chronic Smoke Exposure for Wildland Firefighters. J Occup Environ Hyg. 2004 May:1(5):296-305. doi: 10.1080/15459620490442500 PMID: 15238338

32. Zhang L, Steinmaus C, Eastmond DA, Xin XK, Smith MT. Formaldehyde exposure and leukemia: a new meta-analysis and potential mechanisms. Mutat Res. 2009 MarJun;681(2-3):150-168. doi: 10.1016/.mrrev.2008.07.002.

33. Nordman $\mathrm{H}$, Kesikinen $\mathrm{H}$, Tuppurainen $\mathrm{M}$. Formaldehyde asthma-rare or overlooked?. J Allergy Clin Immunol. 1985 Jan;75(1 Pt 1):91-9. doi: 10.1016/0091-6749(85)90018-1. PMID: 3968333.

34. NaK, Cocker D. Fine organic particle, formaldehyde, acetaldehyde concentrations under and after the influence of fire activity in the atmosphere of Riverside, California. Environ Res 2008:108:7-14. doi:10.1016/j.envres.2008.04.004.

35. Black C, Tesfaigzi Y, Bassein JA, Miller LA. Wildfire smoke exposure and human health: Significant gaps in research for a growing public health issue. Environ Toxicol Pharmacol. 2017:55:186-95. doi: 10.1016/j.etap.2017.08.022 